\title{
Preference for food and water in rats as a function of delay of reward
}

\author{
STEPHANIE S. SMITH and K. EDWARD RENNER \\ University of Illinois, Champaign, Illinois 61820
}

\begin{abstract}
In Experiment I, rats which were both hungry and thirsty were given a choice between a food reward and a water reward. The animals preferred food to water when the reward was delivered immediately, but preferred water to food when a 30-sec delay was imposed in the goalbox before the reward was received. Experiment II replicated the results of the first experiment and showed, in addition, that when the delay was imposed in a separate delay chamber devoid of differential goalbox cues, subjects preferred food to water, similar to the immediate group. The results were discussed in terms of an incentive value process and a competing response hypothesis.
\end{abstract}

Logan and Spanier (1970), using a between-subjects design and a straight alley runway, have found that rats run faster for food than for water when the reward is immediate but run faster for water than food if the reward is delayed. Simply put, delay of water reward is not as detrimental to performance as delay of food reward. Logan and Spanier suggested a competing response explanation for their findings. Specifically, unlearned responses associated with hunger and food reward which are elicited in an anticipatory form interfere with running to a greater extent than those associated with thirst and water reward.

There is, however, an alternative explanation. Renner $(1967,1972)$ has demonstrated a relationship between performance, as assessed by running speed measures, and the relative value of incentives, using preference measures. Typically, those factors associated with faster running speeds are also those factors which have a larger value when their relative worth to the subject is measured.

An incentive-value interpretation applied to Logan and Spanier's results would view the performance differences they found as reflecting the particular value of the food or water reward under the different delay conditions. For such an interpretation to be considered, it must be first demonstrated that a subject will prefer food over water when the reward is immediate, but prefer water to food when the reward is delayed, thus establishing the fact of a differential value of food and water under conditions of immediate and delayed reward. The purpose of the first experiment was to use a simple choice paradigm to determine if the necessary preference relationships would be obtained.

Now at Dalhousie University. Requests for reprints should be sent to K. Edward Renner, Department of Psychology, Dalhousie University, Halifax, N.S., Canada.

\section{EXPERIMENT I}

\section{Method}

Subjects. Twenty naive female hooded rats, 61 days old at the start of the experiment, served as subjects.

Apparatus. The choice apparatus used in the experiment has been described previously (Renner, 1964). It consisted of a narrow $(9 \times 20 \mathrm{~cm})$ startbox, the right and left sides of which were motor-driven overhead storing doors. When raised, these doors allowed the subject access to the left or right delay chamber located immediately adjacent to the left and right sides of the startbox. A center door, which was stored under the apparatus, was raised to force a response if necessary and to confine the subject after a choice to one of the two delay chambers. An additional motor-driven overhead door separated each delay chamber from a goalbox at each end of the apparatus. The delay and goal chambers were each 20 -cm cubes. Mounted at one end of the apparatus was a foodcup intu which a $45-\mathrm{mg}$ pellet could be automatically dispensed. A .4-cc burst of tap water could be delivered through a tube to a small well drilled in a Plexiglas cylinder mounted at the opposite end of the apparatus. For half the subjects, the right side of the apparatus was black and the left side was white; these colors were reversed for the other half of the subjects.

Procedure. The animals were handled for 6 days prior to the start of preliminary training and were made both hungry and thirsty by being placed on a $24-h$ deprivation schedule consisting of $60 \mathrm{~min}$ free access to food and water immediately after their daily trials. On Days 1 and 2 of preliminary training, each animal was placed first in the food goal chamber and then in the water goal chamber, where the appropriate reward was delivered to the subject. On Day 3, for half the animals, both the food and water rewards were delayed for $30 \mathrm{sec}$ after the animal was placed in the goalbox. This procedure was continued, four goal placements per day, for 11 additional days, at which time all subjects were eating and drinking in response to the sound of the food and water mechanisms.

On the first day of experimental trials, each subject was placed in the center startbox and the doors were raised; allowing free access to the right and left sides of the apparatus. When the animal broke the photocell located inside the left or right delay chamber, the subject was considered to have made a choice and the center door was raised, confining the animal to either the right or left side. If the subject chose the left side, the door to the left goal chamber was raised and a three-pellet reward was delivered to the goal cup. A choice to the right, however, resulted in the right goal chamber door's being raised and the delivery of the water 


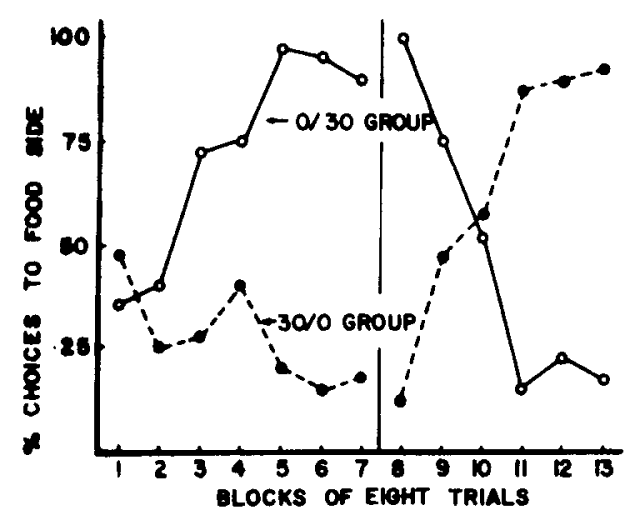

Figure 1. Percentage of free choices to the food side over trials for the group receiving first immediate and then delayed reward (0/30) and for the group receiving first delayed and then immediate reward $(30 / 0)$.

reward. The goal door was closed as soon as the subject entered the goalbox. For half the subjects, both the food and water rewards were dispensed when the animal broke the photocell at the entrance to the goal chamber. For the rest, $30 \mathrm{sec}$ elapsed before the reward was received. All subjects were removed from the apparatus $25 \mathrm{sec}$ after the reward had been received.

Each free-choice trial was balanced by a trial in which the animal was forced to the opposite side of the apparatus by raising only one of the two startbox doors. This insured that at the end of each block of trials all subjects had equal exposure to both alternatives. On the 1 st and 2 nd days of the experiment, two trials per day were run, one free and one forced. For the remainder of the experiment, four trials were run each day. Following Day 15, the conditions were reversed for the two groups so that the immediate reward group was shifted to 30 -sec delay and the 30-sec-delay group was shifted to immediate reward. All animals were then run for an additional 12 days.

\section{Results}

The percentages of free-choice responses to the food side over trials are shown in Figure 1. As can be seen in the left-hand panel, the 0-sec-delay subjects preferred food and the 30 -sec-delay subjects preferred water. The effect was strong and was true of all subjects, $\mathrm{F}(1,18)=88.83, \mathrm{p}<.01$.

The right-hand panel shows the sharp reversal of preference once the immediate reward group was shifted to 30 -sec delay, and the 30-sec-delay group shifted to immediate reward. The interaction term was large, $F(5,90)=50.72, p<.01$, and again the effect was true of all subjects.

\section{Discussion}

A food reward has greater value than a water reward when both are immediate, but less value when both are delayed. Thus, in our study, the relative value of an outcome was associated with the same set of conditions producing differential performance as found by Logan and Spanier (1970).

The consistency of the results using performance and value measures suggests that they share some important feature. On the one hand, it could be that performance measures are best seen as decisions to run faster or more slowly based on incentive processes which reflect the net value of the outcome. Or, on the other hand, as Logan and Spanier suggested, features of the instrumental response process, such as delay engendered responses associated with hunger or eating but not with thirst or drinking, could interfere with running to food. Given our data, these competing responses would also have to be capable of affecting the value of the food.

Both interpretations require some mechanism or means whereby a preference for food or water is differentially affected by waiting. One such possibility is that waiting to eat in the presence of the cues associated with eating evokes preparatory activity, such as salivation, which, without immediate reward, is aversive and thus reduces the net value of the food. In contrast, drinking may require less in the way of preparation.

If this were true, it was reasoned that the effect should occur only if the subjects were delayed in the goalbox, in the presence of the foodcup and the water dish, as they were in the first experiment. But, if the subjects were delayed in a separate chamber, devoid of any cues similar to either goalbox, then the effect should disappear. In this case, there should not be any differential preparatory activity associated with waiting on the food side as compared with waiting on the water side. Therefore, the delay subjects should be similar to the immediate reward subjects.

The second experiment was conducted to test this prediction and to provide a replication of the uiginal study.

\section{EXPERIMENT II}

\section{Method}

Subjects. Eighteen naive female hooded rats, 160 days old at the start of the experiment, served as subjects.

Apparatus. The apparatus was the same as that used in Experiment I.

Procedure. The subjects were divided into three groups of six animals each, all of which were given $40 \mathrm{~min}$ of free access to water and $90 \mathrm{~min}$ of free access to food after the completion of the daily trials. The procedure was otherwise identical with the original study, but with the important difference that a gray delay chamber was introduced on both the left and right sides of the startbox. Thus the choice point was separated from the differential cues of the black goalbox at one end of the apparatus and the white goalbox at the other end. In the original study, the entire arm to the left and right of the start area had been either white or black.

In this experiment, we needed to show that the use of a gray delay chamber did not alter the basic finding. The first two groups therefore replicated the original study. These subjects chose one side or the other with only the gray chamber visible; as soon as the choice was made, the goalbox door was raised and the subject could pass from the gray stem into the goal area. One group was rewarded immediately in the goalbox; the other group, after after $30 \mathrm{sec}$. The third group, in contrast, was delayed in the gray delay chamber for $30 \mathrm{sec}$ after the choice and then given access to the goaibox and to the food or water reward. All subjects were confined to the goalbox after entry and were removed $25 \mathrm{sec}$ after the reward was delivered, as in the first experiment. 


\section{Results}

As can be seen in Figure 2, the original study was replicated. When the delay occurs in the goalboxes, immediate-reward subjects prefer food but delayreward subjects prefer water, $F(1,10)=228, p<.01$. However, when the delay takes place in the separate gray chamber, without distinctive goal-related cues, these subjects prefer food $(p<.02$, sign test), similar to the immediate subjects, although not as strongly in absolute terms, $\mathrm{F}(1,10)=9.30, \mathrm{p}<.05$. The subjects which are delayed in the distinctive goalboxes prefer water $(p<.02$, sign test), thus differing from those delayed in the separate gray chamber, both in direction of preference as well as in an absolute sense, $\mathrm{F}(1,10)=108.75, \mathrm{p}<.01$.

\section{DISCUSSION}

Any theory which presumes to provide an adequate explanation for the empirical results provided by Logan and Spanier's and our experiments must account for the following conditions: (1) Food and water reward have differential effects, depending on whether the reward is immediate or delayed. (2) The effect is seen in both performance and value measures. (3) The differential effect is strong and clear when the delay occurs in the goalbox, but is sharply reduced (Logan \& Spanier, 1970) or small and relative (Experiment II) when the delay is isolated from goal-related cues. Two types of explanations have been suggested, one based on response and one on value processes. Neither provides an adequate or comprehensive theoretical account.

Logan and Spanier's runway procedure, on the one hand, is compatible with a competing response type of explanation, which holds that responses produced by waiting for food, which are later elicited in an anticipatory form, interfere with running to food to a greater extent than those produced by waiting for water interfere with running to water. This view requires that hunger and thirst be seen as qualitatively different in terms of unlearned responses to those drive states, such as a greater variability of motor behavior as a consequence of hunger than of thirst (Petrinovich \& Bolles, 1954). Thus, under delay conditions, thirsty animals would be expected to maintain a greater orientation toward the goal during the delay interval than hungry animals, and the greater number of defay-engendered competing responses associated with hunger would be assumed to interfere with running. Left unclear is how this view should be extended to include the preference data.

Our choice procedure, on the other hand, is com-

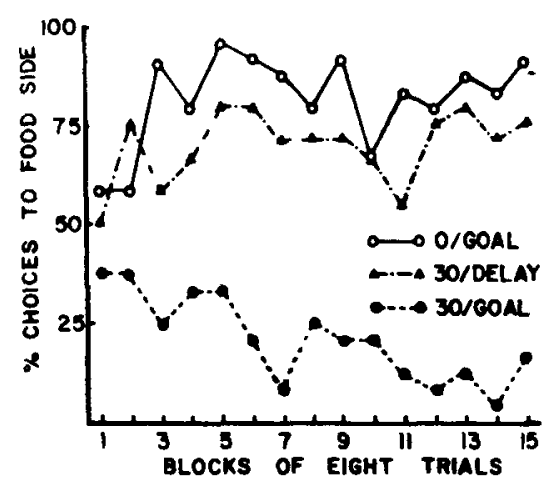

Figure 2. Percentage of free choices to the food side over trials for the group rewarded immediately after entry to the goalbox (0/goal) and for the group rewarded $30 \mathrm{sec}$ after entry to the goalbor (30/goal), thus replicating Experiment I. The percentage of free choices for the group delayed for $30 \mathrm{sec}$ in the delay chamber $(30$ /delay) before receiving the reward is also shown.

patible with an incentive-value type of explanation. Specifically, the choice procedure provides an operational definition of value. When response ontcomes are held constant and when experience with both alternatives is equal, as in our experiments, it is safe to infer that the subject knows the location and temporal distribution of the food reward on one side and the water reward on the other side. Because expectancies are stable, value processes can be observed directly through preference (Irwin, 1971). Left unclear with this account is why waiting in the actual presence of the goal-related cues is necessary for a delayed food reward to lose its relative advantage over water. Some undetermined process specific to hunger or eating must be seen as mediating the differential values observed in our studies. And, with this account, final performance, in terms of running speed, must be assumed to be a direct reflection of these relative values.

At present, there is no suitable empirical basis for viewing the effect either as due to response or value processes, or to some other process which manifests itself in both performance and preference methodologies. The empirical phenomenon is of theoretical interest concerning the need to make qualitative distinctions between motivational processes associated with hunger and thirst, and between response and value processes in the organization of behavior. Theoretical clarification will require the identification of additional variables which may, or do, influence the strength or direction of the differential effects of food and water rewards, such as level of deprivation, or the place of delay confinement, as in our second experiment. 


\section{REFERENCES}

IRwiN, F Intentional behavor and motivation Philadelpha Lippincott. 1971.

Logan, F. A , \& Spanier, D. Relative effect of delay of tood and water reuard. Journal of Comparative and Physiological Psychology, 1970. 72, 102-104.

P'etrinovich, L., \& Bolles, R. Deprivation states and behavioral attributes. Joumal of Comparative and Phy siological Psychology, 1954, 47, 450-453.

Renner, K. E. Conflict resolution and the process of tempora! integration. Psychological Reports, 1964, 15, 423.438.
RFNnek, K E. lemporal integration Ar incentive approach to contlut resolut on. In B A Maher (Ed.). Progress in experimental personaluty research (Vol. 4) New Yotk. Academic Press. 1967. Pp. 127-177.

Renner. K. E. Coherent self-direction and values Annals or the New York Acac'emy of Science, 1972, 193, 175-184.

(Received for publication December 8, 1975; revisior accepted February 23, 1976.) 